\title{
MORBIDADE DA DOENCCA DE CHAGAS. ESTUDO SECCIONAL EM UMA ÁREA ENDÊMICA, VIRGEM DA LAPA, MINAS GERAIS
}

\author{
José Borges Pereira e José Rodrigues Coura
}

De janeiro a abril de 1982 foi desenvolvido um estudo seccional sobre a morbidade da doença de Chagas humana na área urbana do municipio de Virgem da Lapa, nordeste de Minas Gerais, Vale do Jequitinhonha.

A prevalência da infecção chagásica avaliada através da reação de imunofluorescência indireta em sangue colhido em papel de filtro foi de $12,6 \%$, em uma amostra de 2.787 pessoas residentes. $O$ indice da infecção foi mais elevado no grupo de mulheres $(p<0,001)$ em relação ao de homens; aumentou progressivamente com a idade até a quinta década da vida, a partir da qual se estabilizou.

Os exames clínico, eletrocardiográfico e radiológico de 255 chagásicos crônicos, pareados por idade e sexo com igual número de não chagásicos-controles, revelaram as formas clínicas da doença nas seguintes freqüências: 118 (46,3\%) na forma indeterminada, 109 (42,7\%) na cardíaca, 19 (7,5\%) na mista (cardiopatia + megaesôfago) e $9(3,5 \%)$ na digestiva (megaesôfago).

A positividade global em 90 xenodiagnósticos foi de $36,7 \%$, com predomínio entre os pacientes do sexo masculino e na forma clínica indeterminada.

Palavras chaves: Doença de Chagas. Morbidade. Estudo seccional. Cardiopatia. Megaesôfago.

Após a descoberta da "Nova Tripanozomiaze Humana" por Carlos Chagas 17 em território brasileiro, naturalmente se esperava que os estudiosos da época, situados fora de Manguinhos, despertassem e promovessem pesquisas no sentido de, o mais rápido possivel, dimensionar a sua importância mórbida e social. No entanto, cerca de duas décadas se passaram e as significativas contribuições sobre a nova entidade clínica emergiam especificamente dos laboratórios de Manguinhos 1819596061 .

Os estudos sistemáticos sobre a epidemiologia da doença de Chagas, no Brasil, começaram no início dos anos quarenta em Bambui-MG onde foi implantado por Emmanuel Dias e outros pesquisadores um programa de pesquisas e controle da endemia. Inúmeros estudos relacionados com esse programa têm revelado expressivas contribuições sobre a história natural da infeç̧ão humana pelo $T$. cruzi 262728 2930343536 .

Em seguida às observações iniciais obtidas em Bambui, a doença passou a ser referida com maior freqüência em outras áreas endêmicas brasileiras assim como em estudos desenvolvidos em ambiente hospitalar 391011122025384955 .

A partir dos anos cinqüenta a literatura foi enriquecida por importantes contribuições sobre a evolução e o prognóstico da doença de Chagas, em

Departamento de Medicina Tropical do Instituto Oswaldo Cruz.

Financiado pelo CNPq (PIDE) e FINEP.

Recebido para publicação em 12/9/85. especial da cardiopatia crônica 1323474850515354 . No inicio dos anos setenta foi criado o Programa Integrado de Doenças Endêmicas (PIDE), patrocinado pelo $\mathrm{CNPq}$, o qual, além de permitir a ampliação das investigações sobre a tripanosomíase cruzi, procurou uniformizar a metodologia das pesquisas epidemiológicas ${ }^{21}$. Com isso foram ativados os estudos de campo em áreas como: São Felipe ${ }^{39}$ e Castro Alves 4142 na Bahia, Pains-Iguatamal 24 e Virgem da Lapa $^{31} 46$ em Minas Gerais, Mambai16 em Goiás, Oeiras $^{22}$ no Piauí, Sertão da Paraíba ${ }^{4}$ e zona sul do Rio Grande do Sul 2567 .

Com o objetivo de caracterizar aspectos da morbidade da doença de Chagas em uma área endêmica com transmissão da infecção por triatomineos interrompida há pouco mais de dez anos, desenvolvemos o atual estudo seccional que se constitui no segundo realizado em Virgem da Lapa. A casuística envolvida nesse trabalho é parte da tese de mestrado apresentada ao Departamento de Medicina Tropical do Instituto Oswaldo Cruz em dezembro de 1983 por J.B. Pereira.

\section{CASUÍSTICA E MÉTODOS}

O presente trabalho foi desenvolvido de janeiro a abril de 1982 na área urbana do município de Virgem da Lapa, situado no médio Jequitinhonha, nordeste de Minas Gerais, a cerca de $600 \mathrm{~km}$ de Belo Horizonte. Para tal empregamos as normas gerais sobre estudos epidemiológicos da doença de Chagas contidas no relatório técnico no 1 do $\mathrm{CNPq}^{21}$. 
Pereira JB, Coura JR. Morbidade da doença de Chagas. Estudo seccional em uma área endêmica, Virgem da Lapa. Minas Gerais. Revista da Sociedade Brasileira de Medicina Tropical 19:139-148, Jul-Set, 1986

Para dimensionarmos a prevalência da infecção chagásica tentamos alcançar toda a população de 3.400 pessoas residentes maiores de seis meses de idade. Contudo, somente conseguimos colher sangue em papel de filtro neutro de 2.787 pessoas. $O$ grupo não atingido, nessa etapa, constitui-se de pessoas ausentes e das que se recusaram. Todas as amostras de sangue foram submetidas à reação de imunofluorescência indireta segundo Souza e Camargo ${ }^{58}$.

A análise da doença foi realizada em uma amostra de 255 indivíduos soropositivos (chagásicos) pareados por idade e sexo com igual número de indivíduos soronegativos (não chagásicos), distribuidos na Tabela 1. Foram considerados soropositivos ou soronegativos, para efeito de pareamento, os individuos em que os soros apresentavam resultados positivo ou negativo concomitantemente para as técnicas de imunofluorescência indireta ${ }^{14}$, hemaglutinação indireta ${ }^{15}$ e fixação do complemento ${ }^{8}$ para anticorpos contra o $T$. cruzi.

Constituídos os grupos de chagásicos e de não chagásicos (controles), ambos foram submetidos aos exames clínico, eletrocardiográfico e radiológico. Em 90 chagásicos foi aplicado o xenodiagnóstico.

A observação clínica constou da anamnese e do exame físico dirigidos, especialmente, para a identificação das manifestações ligadas aos aparelhos cardiovascular e digestivo, com registro em ficha padronizada para esse tipo de estudo.

OECG foi obtido em repouso, registrando-se as doze derivaçōes clássicas com $D 2$ longo em caso de arritmias. A interpretação dos traçados obedeceu aos critérios da New York Heart Association-NYHA ${ }^{43}$, considerando normais as freqüências de 60 a 120 ciclos/min.

No exame radiográfico obteve-se abreugrafia de $70 \mathrm{~mm}$ em PA e perfil com esôfago contrastado em duas tomadas: uma logo após a ingestão do contraste $e$ a outra um minuto depois ${ }^{32} 37$. Considerou-se cardiomegalia quando o índice cardiotorácico (ICT) era superior a 0,5 . A identificação e classificação do megaesôfago obedeceram aos critérios de Rezende, Oliveira e Lauar ${ }^{56}$, que se resumem em quatro grupos radiológicos: Grupo I - esôfago de calibre aparentemente normal ao exame radiológico, trânsito lento, pequena retenção do contraste; Grupo $I I$ - esôfago com pequeno a moderado aumento de calibre, apreciável retenção do contraste, observadas com freqüência ondas terciárias, associadas ou não à hipertonia do esôfago inferior; Grupo III - esôfago com grande aumento de calibre; hipotonia da porção inferior, atividade motora reduzida ou inaparente, grande retenção do contraste; Grupo IV - dolicomegaesôfago. Esôfago com grande capacidade de retenção, atônico, alongado, dobrando-se sobre a cúpula diafragmática.

Para definir o grau da lesão cardíaca, entre os chagásicos utilizamos os critérios preconizados por peritos da OMS/OPS 44 que através dos exames clínico, eletrocardiográfico è radiológico resumem-na em: Grau I - infecçāo chagásica sem indicações clinicas, radiológicas e eletrocardiográficas de lesão cadíaca; Grau II - infeç̧ão chagásica com sintomatologia moderada ou nula, radiologia normal ou indicação de hipertrofia cardiaca leve e com alterações eletrocardiográficas do tipo: extrassístole ventricular, bloqueio incompleto ou completo do ramo direito do feixe de His, bloqueio incompleto ou completo do ramo esquerdo do feixe de His, bloqueio aurículoventricular incompleto, alterações da repolarização ventricular; Grau III - infeeção chagásica com sintomatologia evidente, hipertrofia cardíaca moderada e alterações eletrocardiográficas do tipo: bloqueio completo do ramo direito do feixe de His com desvio do eixo elétrico médio do QRS para a esquerda, zonas eletricamente inativas, bloqueio aurículo-ventricular completo ou fibrilação ou flutter auricular; Grau IV infecção chagásica com sintomatologia muito pronunciada com insuficiência cardíaca. $O$ estudo radiológico mostra cardiomegalia extrema e o eletrocardiograma indica alterações graves e múltiplas (arritmias com-

Tabela 1 - Distribuiçâo dos pares examinados

\begin{tabular}{|c|c|c|c|c|c|c|}
\hline \multirow{2}{*}{$\begin{array}{l}\text { Grupo } \\
\text { etário }\end{array}$} & \multicolumn{2}{|c|}{ Pares masculinos } & \multicolumn{2}{|c|}{ Pares femininos } & \multicolumn{2}{|c|}{ Total de pares } \\
\hline & $N o$ & $\%$ & $N o$ & $\%$ & $N o$ & $\%$ \\
\hline $6-9$ & 1 & 1,3 & 2 & 1,1 & 3 & $\overline{1,2}$ \\
\hline $10-19$ & 4 & 5,1 & 21 & 11,9 & 25 & 9,8 \\
\hline $20-29$ & 6 & 7,7 & 14 & 7,9 & 20 & 7,8 \\
\hline $30-39$ & 13 & 16,7 & 35 & 19,8 & 48 & 18,8 \\
\hline $40-49$ & 23 & 29,5 & 43 & 24,3 & 66 & 25,9 \\
\hline $50-59$ & 16 & 20,5 & 29 & 16,4 & 45 & 17,6 \\
\hline Mais de 60 & 15 & 19,2 & 33 & 18,6 & 48 & 18,8 \\
\hline Total & 78 & 100,0 & 177 & 100,0 & 255 & 100,0 \\
\hline
\end{tabular}


plexas e graves ou extensas zonas eletricamente inativas).

No xenodiagnóstico aplicado em 90 pacientes chagásicos, empregou-se 40 ninfas de Triatoma infestans do 30 ao 5\% estádios. A leitura ocorreu aos 30 dias após o repasto com a observação microscópica das fezes de cada inseto.

$\mathrm{Na}$ análise estatistica dos dados, além das medidas descritivas, empregamos o teste do quiquadrado, considerando a rejeição da hipótese nula ao nivel de $p<0,05$. Também foi empregado o método de Sheps ${ }^{57}$ na estimativa da proporção de participação do componente etiológico exclusivamente chagásico nas freqüências da cardiopatia e do megaesôfago.

\section{RESULTADOS}

Prevaléncia da infecção. Foi de $12,6 \%$ a proporção de pessoas com a reação de imunofluorescência indireta (IFI) positiva. Observou-se o aumento progressivo da infecção com a idade até a quinta década da vida, a partir da qual ela se estabilizou (Tabela 2). Houve predomínio significativo da infecção no grupo de mulheres em relação ao de homens $(p<0,001)$. Todos os casos de IFI positiva em menores de dez anos foram identificados em pessoas naturais da zona rural do mesmo município que estão residindo na zona urbana por períodos de até três anos. Por outro lado, entre as pessoas nascidas na área urbana, foi de 13 anos a menor idade para a reação de IFI positiva.

Clínica. Considerando o aparelho cardiovascular, $40 \%$ dos chagásicos e $15 \%$ dos controles eram sintomáticos, embora todos se mostrassem em equilibrio funcional, desempenhando suas atividades habituais. Faz-se exceção a um chagásico de 76 anos que se encontrava em franca insuficiência cardíaca congestiva. Entre os sintomas, os mais referidos, em ordem decrescente, foram: palpitações, dispnéia e dor precordial aos esforços, enquanto entre os sinais verificaram-se: distúrbio do ritmo, desdobramento da segunda bulha e desvio do ictus para a linha axilar anterior. Não foi observada diferença nas proporções de hipertensão arterial entre os grupos de chagásicos e controles. Quanto ao aparelho digestivo, $27 \%$ dos chagásicos e $5 \%$ dos controles apresentaram manifestações, sendo a disfagia a mais referida, seguida de pirose, dor esofagiana, regurgitações e obstipação superior a cinco dias. Em sete chagásicos $(2,7 \%)$, de ambos os sexos e maiores de 36 anos, foi diagnosticado fecaloma ao exame físico.

Eletrocardiografia. Considerando todas as alterações registradas, diagnosticou-se ECG anormal em $50,2 \%$ dos chagásicos e em $24,3 \%$ dos controles; indicando um risco relativo 2,1 vezes maior para os chagásicos (Tabela 3). No entanto, ao ser considerado

Tabela 2 - Prevalência da infecção chagásica.

\begin{tabular}{|c|c|c|c|c|c|c|c|c|c|}
\hline \multirow{2}{*}{$\begin{array}{l}\text { Grupo } \\
\text { etário }\end{array}$} & \multicolumn{3}{|c|}{ Masculino } & \multicolumn{3}{|c|}{ Feminino } & \multicolumn{3}{|c|}{ Total } \\
\hline & Exam. & Pos. & $\%$ Pos. & Exam. & Pos. & $\%$ Pos. & Exam. & Pos. & $\%$ Pos. \\
\hline $0-9$ & 359 & 5 & 1,4 & 388 & 3 & 0,8 & 747 & 8 & 1,1 \\
\hline $10-19$ & 316 & 7 & 2,2 & 494 & 31 & 6,3 & 810 & 38 & 4,7 \\
\hline $20-29$ & 104 & 8 & 7,7 & 201 & 22 & 10,9 & 305 & 8 & 9,8 \\
\hline $30-39$ & 101 & 15 & 14,8 & 174 & 53 & 30,5 & 275 & 68 & 24,7 \\
\hline $40-49$ & 102 & 30 & 29,4 & 164 & 56 & 34,1 & 266 & 86 & 32,3 \\
\hline $50-59$ & 56 & 20 & 35,7 & 106 & 32 & 30,2 & 162 & 52 & 32,1 \\
\hline Mais de 60 & 80 & 24 & 30,0 & 142 & 46 & 32,4 & 222 & 70 & 31,5 \\
\hline Total & 1118 & 109 & 9,7 & 1669 & 243 & 14,6 & 2787 & 352 & 12,6 \\
\hline
\end{tabular}

Tabela 3 - Estudo eletrocardiográfico em 255 pares de chagásicos e não chagásicos

\begin{tabular}{lccccc}
\hline \multirow{2}{*}{ Eletrocardiograma } & \multicolumn{2}{c}{ Grupo chagásico } & & \multicolumn{2}{c}{ Grupo não chagásico } \\
\cline { 2 - 3 } \cline { 5 - 6 } & $N o$ & $\%$ & & \multirow{2}{*}{$\%$} & $\%$ \\
\hline Normal & 127 & 49,8 & & 193 & 75,7 \\
Alterado* & 128 & 50,2 & & 62 & 24,3 \\
\hline Total & 255 & 100,0 & & 255 & 100,0 \\
\hline
\end{tabular}

* Considerando todas as alterações. 
anormal o ECG que apresentava alterações do tipo: BRD III isolado ou associado ao HBAE, extrassístole ventricular freqüente uni ou polifocal, bloqueio auriculo-ventricular e zona eletricamente inativa, as proporçōes obtidas foram, respectivamente, $33,3 \%$ e $2,4 \%$, elevando o risco para 13,7 .

Embora a freqüência de ECG anormal, entre os chagásicos, tenha sido mais elevada no grupo de homens em relação ao de mulheres, não houve diferença significativa $(p>0,05)$. A Figura 1 mostra o comportamento das proporções de ECG alterados em

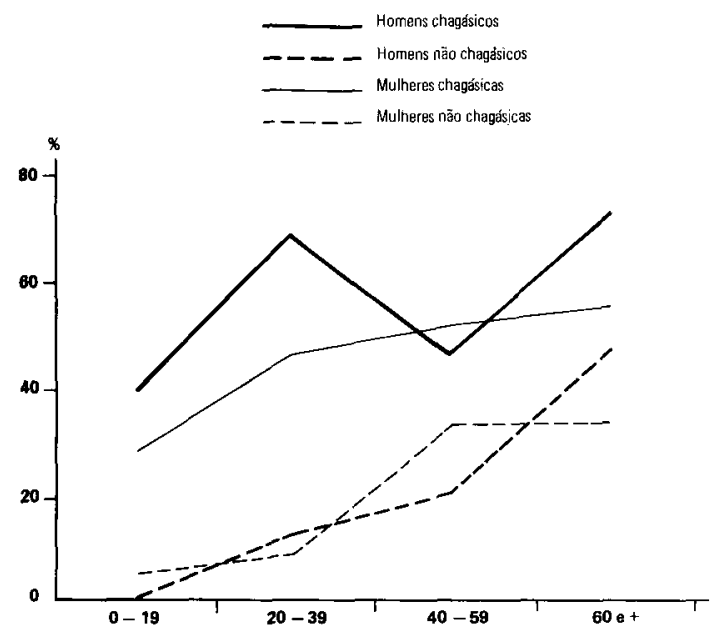

Figura 1 - Proporçôes de ECG alterados em chagásicos e não chagásicos em função do grupo etário. relação aos grupos etários; destacando-se os niveis crescentes com a idade tanto em chagásicos como nos controles e a significativa queda no grupo de homens chagásicos dos 40 aos 59 anos.

$\mathrm{Na}$ Tabela 4 estão assinaladas as proporções de todas as alterações eletrocardiográficas identificadas na amostra estudada. Dos 47 casos de BRD III, 37 $(78,7 \%)$ estavam associados ao HBAE e $35(74,5 \%)$ apresentaram, ao exame fisico, desdobramento acentuado da segunda bulha cardiaca. Todos os chagásicos com extrassistoles ventricular, ao ECG, referiram palpitações e em $86,5 \%$ deles o exame fisico revelou distúrbio do ritmo. Ao compararmos a ocorrencia de BRD III e de extrassistole ventricular em relação à idade dos chagásicos, verificamos que $68,1 \%$ dos casos de BRD III ocorreram em pacientes com idade abaixo de 40 anos enquanto que $56,0 \%$ dos casos de extrassistoles ocorreram em maiores de 40 anos.

Radiologia. Foram estudados apenas 251 pares nessa etapa, em decorrência de quatro mulheres grávidas não terem sido submetidas às radiografias. Foram identificados 23 casos $(9,2 \%)$ de cardiomegalia entre os chagásicos e cinco casos $(2,0 \%)$ entre os controles; não houve diferença significativa quanto ao sexo em ambos os grupos. A frequeência de cardiomegalia aumentou com a idade dos pacientes, sendo diagnosticada a partir da terceira década da vida entre os chagásicos e a partir da quinta década entre os controles. Todos os pacientes com cardiomegalia eram sintomáticos e tinham os ECG alterados, especialmente por extrassístole ventricular (15 casos), BRD III + HBAE (nove casos), distúrbio primário da repolarização ventricular (sete casos), zona eletrica-

Tabela 4 -Alterações eletrocardiográficas em 255 pares de chagásicos e não chagásicos.

\begin{tabular}{|c|c|c|c|c|c|}
\hline \multirow{2}{*}{ Alteraçōes } & \multicolumn{2}{|c|}{ Grupo chagásico } & \multicolumn{2}{|c|}{ Grupo não chagásico } & \multirow{2}{*}{$\begin{array}{l}\text { Excesso de } \\
\text { risco }\end{array}$} \\
\hline & $N .^{o}$ & $\%$ & $N .^{o}$ & $\%$ & \\
\hline Bloqueio completo do ramo direito (BRD III) & 47 & 18,4 & 2 & 0,8 & 17,6 \\
\hline Distúrbio primário da repolarização ventricular & 46 & 18,0 & 28 & 11,0 & 7,0 \\
\hline Extrassistole ventricular freqüente** & 37 & 14,5 & 2 & 0,8 & 13,7 \\
\hline Hemibloqueio anterior esquerdo (HBAE) & 37 & 14,5 & 3 & 1,2 & 13,3 \\
\hline Bloqueio auriculo-ventricular incompleto (BAV I) & 16 & 6,3 & - & - & 6,3 \\
\hline Zona eletricamente inativa & 14 & 5,5 & 2 & 0,8 & 4,7 \\
\hline Extrassistole ventricular isolada & 13 & 5,1 & 7 & 2,7 & 2,4 \\
\hline Baixa voltagem do $Q R S$ & 7 & 2,7 & 10 & $3,9^{\prime}$ & $-1,2$ \\
\hline Extrassístole supraventricular & 7 & 2,7 & 2 & 0.8 & 1,9 \\
\hline Bloqueio incompleto do ramo direito (BRW I) & 5 & 2,0 & 1 & 0,4 & 1,6 \\
\hline Bradicardia sinusal & 5 & 2,0 & 4 & 1,6 & 0,4 \\
\hline Fibrilação auricular & 3 & 1,2 & - & - & 1,2 \\
\hline Bloqueio completo do ramo esquerdo (BRE III) & 3 & 1,2 & 1 & 0,4 & 0,8 \\
\hline Sobrecarga auricular esquerda & 3 & 1,2 & 2 & 0,8 & 0,4 \\
\hline Taquicardia sinusal & 3 & 1,2 & 5 & 2,0 & $-0,8$ \\
\hline Sobrecarga ventricular esquerda & 2 & 0,8 & 7 & 2,7 & $-1,9$ \\
\hline Bloqueio auriculo-ventricular incompleto (BAV II) & 1 & 0,4 & - & - & 0,4 \\
\hline Bloqueio incompleto do ramo esquerdo (BRE I) & 1 & 0,4 & - & - & 0,4 \\
\hline Sobrecarga auricular direita & 1 & 0,4 & - & - & 0,4 \\
\hline
\end{tabular}

\footnotetext{
" porcentagem mais de duas por min.
} 
Pereira JB, Coura JR. Morbidade da doença de Chagas. Estudo seccional em uma área endêmica, Virgem da Lapa, Minas Gerais. Revista da Sociedade Brasileira de Medicina Tropical 19:139-148, Jul-Set, 1986

mente inativa (quatro casos) e fibrilação auricular (dois casos).

Megaesôfago foi identificado em 28 pacientes chagásicos $(11,2 \%)$ e em dois não chagásicos $(0,8 \%)$. De acordo com a distribuição assinalada na Tabela 5 , observa-se que o diagnóstico de "mega" entre os chagásicos ocorreu a partir da terceira década da vida enquanto no grupo controle o foi a partir dos 60 anos. Quanto ao sexo, a prevalência foi de $17,9 \%$ entre os homens e de $8,1 \%$ entre as mulheres com $\mathbf{p}<0,05$. Em relação ao grupo radiológico, predominou o grupo I sobre os demais, nâo sendo evidenciado ne-

Tabela 5-Distribuição do megaesôfago em 251 chagásicos, de acordo com o grupo etário.

\begin{tabular}{lccc}
\hline Grupo etário & $\begin{array}{c}\text { Exami- } \\
\text { nados }\end{array}$ & Mega. & \% Mega. \\
\hline $6-19$ & 28 & - & - \\
$20-39$ & 66 & 6 & 9,1 \\
$40-59$ & 109 & 11 & 10,1 \\
Mais de 60 & 48 & 11 & 22,9 \\
\hline Total & 251 & 28 & 11,2 \\
\hline
\end{tabular}

nhum caso de dolicomegaesôfago (grupo IV) na amostra examinada (Tabela 6). Entre os 28 pacientes chagásicos com "mega", $25(89,3 \%)$ apresentaram queixa de disfagia e, o restante, todos do grupo I, não a referiu. A presença de ECG alterado foi revelada em 19 (67,9\%) desses casos de megaesôfago.

Prevalência da cardiopatia crónica chagásica. Com base nos exames clinico, eletrocardiográfico e radiológico ficou caracterizada a cardiopatia em 128 pacientes $(50,2 \%)$, distribuidos na Tabela 7 de acordo com o grau de comprometimento miocárdico. Destaca-se a maior frequeuência da cardiopatia nos graus III e IV entre os homens em relação às mulheres. Todos os casos de cardiopatia identificados no grupo controle foram classificados como do grau II.

Formas clínicas da doença de Chagas. Diante da metodologia empregada para o diagnóstico da doença verificou-se as seguintes proporçōes para as formas clinicas: 118 pacientes $(46,3 \%)$ na forma indeterminada, $109(42,7 \%)$ na cardíaca, $9(3,5 \%)$ na digestiva (megaesôfago) e $19(7,5 \%)$ na mista (cardiopatia + megaesôfago) (Tabela 8). A forma clinica indeterminada predominou entre as mulheres e as demais formas clínicas entre os homens sem, no entanto, atingir nivel de significância. Observou-se que à redução da forma indeterminada se segue o aumento

Tabela 6 - Distribuição do megaesôfago de acordo com o grupo radiologico.

\begin{tabular}{|c|c|c|c|c|}
\hline \multirow{2}{*}{ Megaesôfago } & \multicolumn{2}{|c|}{ Grupo chagásico } & \multicolumn{2}{|c|}{ Grupo nâo chagásico } \\
\hline & $N o$ & $\%$ & $N o$ & $\%$ \\
\hline Grupo I & 19 & 67,9 & 2 & 100,0 \\
\hline Grupo II & 6 & 21,4 & - & - \\
\hline Grupo III & 3 & 10,7 & - & - \\
\hline Grupo IV & - & - & - & - \\
\hline Total & 28 & 100,0 & 2 & 100,0 \\
\hline
\end{tabular}

Tabela 7 - Grau da cardiopatia crônica chagásica(*) em 255 pacientes, em relação ao sexo

\begin{tabular}{|c|c|c|c|c|c|c|}
\hline \multirow{2}{*}{$\begin{array}{l}\text { Grau de } \\
\text { cardiopatia }\end{array}$} & \multicolumn{2}{|c|}{ Masculino } & \multicolumn{2}{|c|}{ Feminino } & \multicolumn{2}{|c|}{ Total } \\
\hline & $N o$ & $\%$ & $N o$ & $\%$ & $N o$ & $\%$ \\
\hline I & 34 & 43,6 & 93 & 52,5 & 127 & 49,8 \\
\hline II & 27 & 34,6 & 67 & 37,9 & 94 & 36,9 \\
\hline III & 15 & 19,2 & 17 & 9,6 & 32 & 12,5 \\
\hline IV & 2 & 2,6 & - & - & 2 & 0,8 \\
\hline Total & 78 & 100,0 & 177 & 100,0 & 255 & 100,0 \\
\hline
\end{tabular}

(*) De acordo com os critérios OPS/OMS (1974) 
Pereira JB, Coura JR. Morbidade da doença de Chagas. Estudo seccional em uma área endêmica, Virgem da Lapa, Minas Gerais. Revista da Sociedade Brasileira de Medicina Tropical 19:139-148, Jul-Set, 1986

Tabela 8 -Formas clínicas da doença de Chagas em 255 pacientes, de acordo com o sexo.

\begin{tabular}{|c|c|c|c|c|c|c|}
\hline \multirow{2}{*}{ Formas clínicas } & \multicolumn{2}{|c|}{ Masculino } & \multicolumn{2}{|c|}{ Feminino } & \multicolumn{2}{|c|}{ Total } \\
\hline & $N: o$ & $\%$ & $N . o$ & $\%$ & $N o$ & $\%$ \\
\hline Indeterminada & 29 & 37,2 & 89 & 50,3 & 118 & 46,3 \\
\hline Cardíaca & 35 & 44,9 & 74 & 41,8 & 109 & 42,7 \\
\hline Digestiva (megaesôfago) & 5 & 6,4 & 4 & 2,3 & 9 & 3,5 \\
\hline $\begin{array}{l}\text { Mista (cardiopatia }+ \text { mega- } \\
\text { esôfago) }\end{array}$ & 9 & 11,5 & 10 & 5,6 & 19 & 7,5 \\
\hline Total & 78 & 100,0 & 177 & 100,0 & 255 & 100,0 \\
\hline
\end{tabular}

das formas determinadas, especialmente da cardíaca. Através da Figura 2 pode-se verificar que, dentro dos métodos de investigação empregados neste trabalho, a forma cardiaca ocorreu a partir da segunda década da vida e às que envolvem megaesôfago, a partir da quarta década.

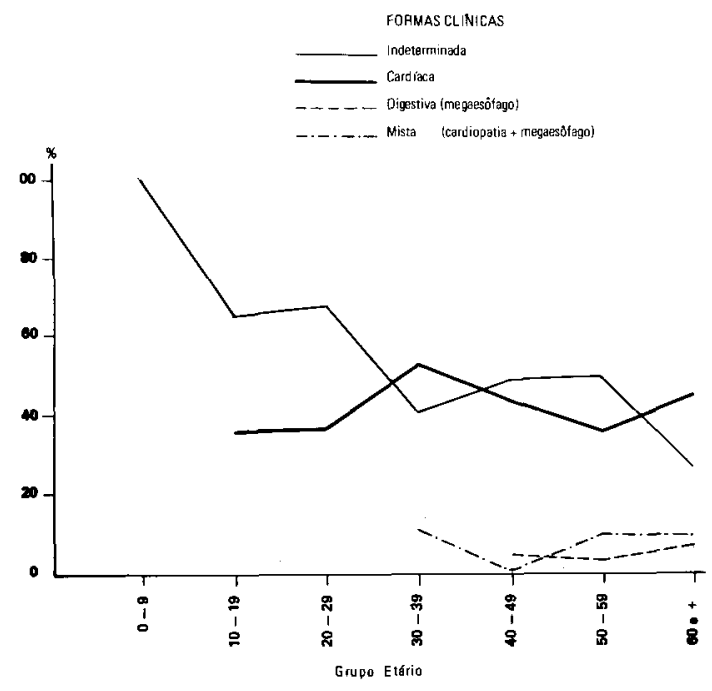

Figura 2 - Proporçes das formas clinicas da doença de Chagas, em funçâo do grupo etário.
Xenodiagnóstico. Um grupo de chagásicos crônicos com idade média de 38 anos foram submetidos ao "xeno", entre eles 25 homens e 65 mulheres. A positividade global dos exames foi de $36,7 \%$ ( 33 pacientes). Treze homens $(52,0 \%)$ e 20 mulheres $(30,8 \%)$ tiveram seus exames positivos. Quanto à idade, os resultados estão assinalados na Tabela 9. Houve maior freqüência de positividade nos pacientes da forma clinica indeterminada em relação aos da forma cardiaca, embora sem significação estatística $(p>0,05)$.

\section{DISCUSSÃO}

Apesar das dificuldades inerentes ao trabalho de campo envolvendo a doença de Chagas, a amostra studada foi considerada representativa da população residente na área em questão.

A prevalência da infecção chagásica assinalada neste inqüérito foi semelhante a descrita por Dubois ${ }^{31}$, há seis anos, na mesma região, empregando a mesma metodologia. Acreditamos que tal observação tenha como fator importante a migração de individuos da zona rural para a urbana, mantendo com isso a populaçãdo de chagásicos. Também deve ser considerado o pequeno intervalo de tempo entre os dois estudos seccionais, visto que a infecção humana pelo $T$. cruzi se caracteriza vela evolução longa que pode durar toda a vida do paciente.

Tabela 9 - Distribuição dos xenodiagnósticos de acordo com o grupo etário.

\begin{tabular}{lccc}
\hline Grupo etário & Examinados & Positivos & \% positivos \\
\hline $6-19$ & 12 & 5 & 41,7 \\
$20-39$ & 25 & 11 & 44,0 \\
$40-59$ & 40 & 11 & 27,5 \\
Mais de 60 & 13 & 6 & 46,1 \\
\hline Total & 90 & 33 & 36,7 \\
\hline
\end{tabular}


A literatura apresenta um número expressivo de trabalhos sobre a prevalência da infecção chagásica humana, nos quais são registrados valores que oscilam entre 4 e 54\%4 52, que no nosso entender dependem tanto das características epidemiológicas das áreas como das diferenças metodológicas.

A maioria dos inquéritos realizados em áreas endêmicas revela maior indice de infecção entre as mulheres em relação aos homens, do mesmo modo que o observado nos estudos desenvolvidos em Virgem da Lapa ${ }^{31}$ 45. Porém não há dados suficientes para que se possa atribuir às mulheres maior susceptibilidade à infecção pelo $T$. cruzi. Outro achado comum que se verifica nesses trabalhos é o crescimento progressivo da prevalência da infecção com a idade das pessoas até a quinta década da vida, a partir da qual são assinaladas diferentes variações no comportamento da curva de proporções. Tais características podem ser atribuidas ao aumento do risco de infecção com a idade do individuo e as diferenças regionais quanto à morbimortalidade da doença chagásica.

$A$ ausência de triatomineo $v$ de criança menor de seis anos infectada na área urbana de Virgem da Lapa, identificadas por Dubois, há seis anos, o levou a afirmar que estava interrompida a transmissão da infecção por tais vetores. No desenvolvimento do atual estudo confirmaram-se tais achados que admitimos dependerem fundamentalmente da eficácia das medidas ao combate dos triatomíneos, através da aplicação de BHC e da melhoria habitacional, desencadeadas a partir do inicio dos anos setenta.

$O$ exame clinico tem-se mostrado de grande importância na indicação da doença de Chagas, tanto no envolvimento cardiovascular como no digestivo. A totalidade dos estudos comparativos realizados em áreas endêmicas revela sıgniticativo predominio das manifestações clínicas entre os chagásicos em relação aos não chagásicos. A sensibilidade desse exame pode ser caracterizada, neste trabalho, através de resultados como: a presença de sintomatologia em pacientes ainda com o ECG e radiografias normais e o elevado percentual de concordância entre a sintomatologia e os resultados de alterações eletrocardiográficas e radiológicas.

No exame eletrocardiográfico, importante revelador da lesão miocárdica, verificou-se em nossa casuistica que cerca de $50 \%$ dos chagásicos apresentaram indicações de cardiopatía, expressando em relação ao grupo controle, um risco duas vezes maior de apresentá-las, considerando todas as anormalidades no traçado. Contudo esse risco aumentou para treze vezes mais ao ser consideradas somente alterações mais específicas da cardiopatia crônica chagásica; traduzindo o elevado nivel de participação da infeç̧ão pelo $T$. cruzi no determinismo da lesão cardiaca nessa área.
Os estudos sobre a ocorrência da cardiopatia crônica chagásica em outras áreas endêmicas, através do ECG, têm mostrado diferenças expressivas nos resultados, revelando ser a doença de Chagas uma entidade clínico-epidemiológica bastante heterogênea. Assim é que recente publicação de Macêdo e $\operatorname{cols}^{40}$, sobre o inquérito eletrocardiográfico nacional, observa diferenças significativas nas proporçōes de cardiopatia entre os chagásicos e controles nas áreas endêmicas de Minas Gerais, Goiás, Bahia, Paraná, Piaui, Paraiba e Pernambuco, além das próprias diferenças nas freqüências da cardiopatia chagásica entre as amostras de cada estado. Por outro lado, nesse mesmo estudo, os autores não encontraram diferença nas taxas de cardiopatia de chagásicos e controles dos estados de Sergipe, Alagoas e Rio Grande do Sul.

Quanto à gravidade da cardiopatia, chama atenção o fato de que mesmo em áreas onde não há diferença quanto às proporções globais entre chagásicos e não chagásicos, encontra-se uma predominância dos casos graves entre os chagásicos 245 . Dado esse também importante quando se refere ao sexo dos pacientes, visto que estudos nos quais não se revelaram, em termos globais, diferenças nos percentuais da cardiopatia, esta se mostra com maior proporção de casos graves entre os homens, para o que não dispomos de explicação consistente. Todos os estudos clínico-epidemiológicos desenvolvidos mesmo em áreas endêmicas distintas revelam que a prevalência da cardiopatia crônica chagásica aumenta com a idade dos pacientes, achado que, provavelmente, indica a necessidade de um longo periodo de infecção para que se expressem as conseqüências mórbidas dessa relação hospedeiro-parasito. Embora não se deva deixar de considerar nesses individuos a possivel inflüência de outros fatores, não relacionados à infecção chagásica, no determinismo da patologia cardíaca e/ou digestiva.

As alterações eletrocardiográficas evidenciadas nesse tipo de estudo, além de indicadoras da cardiopatia, dimensionam a sua gravidade. Quando associadas à cardiomegalia expressam um péssimo prognóstico 2936 .

A frequiência de megaesôfago em nossa casuistica foi de $11,2 \%$ entre os chagásicos. Esse valor está contido dentro dos assinalados em outras áreas endêmicas do Brasil, os quais oscilam entre 6 e 13\%, demonstrando com isso não só a sua presença aonde foi pesquisado como também a sua menor ocorrência em relação à cardiopatia.

$O$ predominio de megaesôfago entre os homens chagásicos em comparação com as mulheres ratifica a observação feita no primeiro estudo seccional, há seis anos, na mesma área ${ }^{31}$. Porém há estudos de outras áreas que não referem tal diferença. 
Pereira JB, Coura JR. Morbidade da doença de Chagas. Estudo seccional em uma área endêmica, Virgem da Lapa, Minas Gerais. Revista da Sociedade Brasileira de Medicina Tropical 19:139-148, Jul-Set, 1986

Diante da observação de que o megaesôfago foi diagnósticado a partir da terceira década entre os chagásicos, enquanto o foi após os sessenta anos entre os controles, acreditamos que a precocidade de sua apresentação nos chagásicos se deva fundamentalmente a participação do componente etiológico chagásico. Por outro lado, os casos revelados em idosos controles, podem ser atribuidos a participação do componente desnervação fisiológica, conforme assinala Koeberle ${ }^{33}$.

A associação de esofagopatia (megaesôfago) à cardiopatia em chagásicos tem sido assinalada em diversos trabalhos realizados tanto em áreas endêmicas como em ambiente hospitalar. No atual estudo observou-se um percentual de associação de $67,8 \%$; caracterizando a multiplicidade de manifestaçōes patológicas que pode ocorrer em um paciente infectado pelo $T$. cruzi.

Dentro da classificação das formas clínicas da doença de Chagas observou-se que a forma indeterminada apresentou-se com freqüência discretamente superior à cardiaca, seguida por valores bem menores para as formas mista e digestiva, a semethança da quase totalidade dos estudos realizados em outras áreas endêmicas com amostras probabilísticas. Também em acordo com os demais trabalhos dessa natureza, verificamos que a redução da taxa da forma indeterminada é acompanhada do aumento da taxa das formas determinadas, com a identificação mais precoce da cardiopatia em relação à esofagopatia.

A positividade global do xenodiagnóstico em nossa amostra foi semelhante a encontrada há seis anos na mesma área ${ }^{31}$. Comparar os resultados deste trabalho e os demais referidos na literatura é extremamente dificil, diante das expressivas diferenças quanto às técnicas de aplicação e leitura dos exames. Contudo podemos citar que há percentuais que vão desde 24 até $68 \% 16$. Além do que, mesmo com diferenças metodológicas, há evidências comuns dentro desses trabalhos, por exemplo: maior positividade do "xeno" nos pacientes masculinos, da forma indeterminada e nos extremos da vida, as quais reclamam de explicações consistentes.

\section{SUMMARY}

From January to April 1982 a cross sectional study of the morbidity of human Chagas' disease was carried out in the urban area of the municipality of Virgem da Lapa, in Northeast Minas Gerais State, in the Valley of Jequitinhonha.

The prevalence of the chagasic infection evaluated by the indirect immunofluorescent test in blood collected on filter paper was $12.6 \%$ in 2.787 samples. The infection rate was higher in women than in men $(p<0.001)$ and the general prevalence of the infection increased progressively up to the fifth decade after which it stabilised.
The clinical, electrocardiographical and radiological examinations in 255 chronic chagasics paired by age and sex with the same number of nonchagasic persons from the same area, showed the following clinical forms in chagasics: 118 (46.3\%) with the indeterminate form, $109(42.7 \%)$ with the cardiac form, 19 (7.5\%) mixed form (cardiac and megaesophagus), and 9(3.5\%) with megaesophagus.

Xenodiagnoses were positive in $36.7 \%$ of the cases, with a predominance in males with the indeterminate clinical form.

Key words: Chagas' disease. Morbidity. Cross-sectional study. Cardiopathy. Megaesophagus.

\section{AGRADECIMENTOS}

Ao Prof. Joffre Rezende pela interpretação das abreugrafias e aos cardiologistas Nei Annunziato e Elcio Pires pela colaboração na interpretação dos eletrocardiogramas.

\section{REFERÊNCIAS BIBLIOGRÁFICAS}

1. Abreu LL. Doença de Chagas: estudo da mortalidade no Município de Pains, Minas Gerais. Tese de Mestrado. Universidade. Federal do Rio de Janeiro, RJ, 1977.

2. Alcantara A, Baruffa G, Aquino Neto JO, Olintho A, Savoldi T, Lassen C. Epidemiologia da doença de Chagas no RS - Revisão de pacientes após 10 anos de evolução. I - Metodologia. II - Grupo chagásico. III - Grupo controle. In: Resumo XIX Congresso da Sociedade Brasileira de Medicina Tropical, Rio de Janeiro, p. 35-36, 1983.

3. Alecrim WD, Castro CN, Rezende JM, Macêdo V, Prata A. Estudo da dinâmica esofágica entre duas áreas endêmicas da doença de Chagas. In: Resumo XIII Congresso da Sociedade Brasileira de Medicina Tropical, Brasília, p. 24, 1977.

4. Arruda Jr ER. Estudo sobre a doença de Chagas nos municípios de Aguiar e Boqueirào, Vale do Piancó, Estado da Paraíba. Tese de Mestrado. Universidade Federal do Rio de Janeiro, RJ, 1981.

5. Baruffa G, Aquino Neto JO, Alcantara A, Bettini VN, Bertinetti ES. Dados preliminares de inquérito sorológico e eletrocardiográfico para a doença de Chagas em populações rurais não selecionadas da zona Sul do Rio Grande do Sul. Revista da Sociedade Brasileira de Medicina Tropical 6: 362-363. 1972.

6. Baruffa G, Alcantara A. Prevalência sorológica da doença de Chagas em cinco municípios da zona Sul do Rio Grande do Sul. Revista do Instituto de Medicina Tropical de São Paulo 16: 140-144. 1974.

7. Baruffa G. Prevalência sorológica da doença de Chagas e correlação sorológica-eletrocardiográfica em populações nảo selecionadas do municipio de Encruzilhada do Sul do Rio Grande do Sul. Revista da Sociedade de Medicina Tropical 9: 95-104, 1975.

8. Batista SM, Santos UM. Antígeno metílico de cultura de Schizotrypanum cruzi. Hospital 56: 1045-1051, 1959. 
Pereira JB, Coura JR. Morbidade da doença de Chagas. Estudo seccional em uma área endêmica, Virgem da Lapa, Minas Gerais. Revista da Sociedade Brasileira de Medicina Tropical 19:139-148, Jul-Set, 1986

9. Benchimol AB, Schlesinger P, Cotrim MR. A cardiopatia chagásica crônica observada na cidade do Rio de Janeiro. Estudo de 32 casos. Medicina-Cirurgia-Farmácia 2/3: 5-30, 1954.

10 Benchimol AB. O eletrocardiograma na miocardite chagásica crônica. Boletim da Academia Nacional de Medicina 133: 9-51, 1962.

11. Brant TC, Laranja FS, Bustamante FM, Mello AL. Dados sorológicos e eletrocardiográficos obtidos em população não selecionada de zonas endêmicas de doença de Chagas no Estado do Rio Grande do Sul. Revista Brasileira de Malariologia e Doenças Tropicais 9: 141-148, 1957.

12. Brasil A. A mutabilidade eletrocardiográfica na cardiopatia chagásica crônica. Revista da Associação Médica de Minas Gerais 4: 149-152, 1953.

13. Brasil A. Evolução e prognóstico da doença de Chagas. Arquivos Brasileiros de Cardiologia 18: 365-380, 1965.

14. Camargo ME. Fluorescent antibody test for the serodiagnosis. Technical modification employing preserved culture forms of Trypanosoma cruz $i$ in slide test. Revista do Instituto de Medicina Tropical de São Paulo 8: 227-34, 1966.

15. Camargo ME, Shimizu SH, Siqueira GVR. Hemagglutination with preserved, sensitized cells, a practical test for routine serologic diagnosis of American Trypanosomiasis. Revista do Instituto de Medicina Tropical de São Paulo 15: 81-85, 1973.

16. Castro $\mathrm{CN}$. Influência da parasitemia no quadro clínico da doença de Chagas. Revista de Patologia Tropical 9: 73-136, 1980.

17. Chagas C. Nova tripanozomiaze humana. Estudo sobre a morfologia e o ciclo evolutivo do Schizotrypanum cruzi, n. gen., n. sp. ajente etiolójico de nova entidade mórbida do homem. Memórias do Instituto Oswaldo Cruz 1: 159-218, 1909.

18. Chagas C. Tripanosomiase americana: forma aguda da moléstia. Memórias do Instituto Oswaldo Cruz 8: 37-60, 1916.

19. Chagas C, Villela E. Forma cardíaca da tripanosomiase americana. Revista de Biologia e Higiene 5: 5-61, 1922.

20. Chapadeiro $E$. Histopatologia cardiaca da forma indeterminada da doença de Chagas. Anais do Congresso Internacional Sobre Doença de Chagas, Rio de Janeiro, 1979.

21. Conselho Nacional de Desenvolvimento Cientifico e Tecnológico - CNPq. Epidemiologia da doença de Chagas. Objetivos e métodos dos estudos longitudinais. Relatório Técnico n: 1, 1974.

22. Correia-Lima FG. Doença de Chagas no Municipio de Oeiras - PI. Estudo seccional nas localidades de Colonia e Oitis. Tese de Mestrado. Universidade Federal do Rio de Janeiro, 1976.

23. Coura JR. Contribuição ao estudo da doença de Chagas no Estado da Guanabara. Revista Brasileira de Malariologia e Doenças Tropicais 18: 9-98, 1966.

24. Coura JR, Abreu LL, Dubois LG, Correia-Lima FG, Willcox HPF, Figueiredo PZ. Estudo seccional sobre a doença de Chagas em 3 diferentes áreas endèmicas no Brasil. Anais do Congresso Internacional Sobre Doença de Chagas p. 41, 1979.
25. Cunha GP, Oliveira PF. Sindromes clinicas na cardiopatia chagásica crônica. Hospital 57: 71-93, 1960.

26. Dias E. Acerca de 254 casos de doença de Chagas comprovados em Minas Gerais. Brazil Médico 60: 41-44, 1946.

27. Dias JCP. Prevalência da doença de Chagas entre crianças da zona rural de Bambui-MG, após ensaio profilático. Revista Brasileira de Malariologia e Doenças Tropicais 19: 135-159, 1967.

28. Dias JCP, Kloetzel K. The prognostic value of the electrocardiographic features of chronic Chagas'disease. Revista do Instituto de Medicina Tropical de São Paulo 10: 158-162, 1968.

29. Dias JCP. Doença de Chagas em Bambuí, Minas Gerais, Brasil. Estudo clínico epidemiológico a partir da fase aguda, entre 1940 e 1982 . Tese de Doutorado. Universidade Federal de Minas Gerais, 1982.

30. Dias JCP, Camacho LAB, Silva JC, Magalhães JS, Krieger $H$. Esofagopatia chagásica na área endêmica de Bambui-MG, Brasil. Revista da Sociedade Brasileira de Medicina Tropical 16: 46-47, 1983.

31. Dubois LG. Morbidade da doença de Chagas. Estudo seccional em uma área endemica. Tese de Mestrado. Universidade Federal do Rio de Janeiro, 1977.

32. Godoy RA, Vieira CB. Diagnóstico da esofagopatia chagásica crônica assintomática nảo ectásica. Revista Goiana de Medicina 9: 117-124, 1963.

33. Koeberle F. Patologia da forma digestiva. Anais do Congresso Internacional sobre Doença de Chagas, 1979.

34. Kloetzel K, Dias JCP. Mortality in Chagas'disease: life table for the period 1949-1967 in an unselected population. Revista do Instituto de Medicina Tropical de São Paulo 10: 5-8, 1968.

35. Laranja FS, Dias E, Nóbrega GC. Clinica e terapêutica da doença de Chagas. Revista Brasileira de Medicina 5: 26p. 1948.

36. Laranja FS, Dias E, Nóbrega GC, Miranda A. Chagas'disease. A clinical, epidemiologic and pathologic study. Circulation 14: 1035-1060, 1956.

37. Lauar KM, Oliveira R, Rezende JM. Valor do tempo de esvaziamento esofagiano ne diagnóstico da esofagopatia chagásica (prova de retenção). Revista Goiana de Medicina 5: 97-102, 1959.

38. Lopes ER, Chapadeiro E, Almeida HO, Rocha A. Contribuição ao estudo da anatomia patológica dos coraçōes de chagásicos falecidos subitamente. Revista da Sociedade Brasileira de Medicina Tropical 9: 269-282, 1975.

39. Macêdo $V$. Influência da exposição à reinfecção na evolução da doença de Chagas (estudo de cinco anos). Revista de Patologia Tropical 5: 33-116, 1976.

40. Macêdo V, Prata A, Rodrigues da Silva G, Castilho E. Prevalência de alterações eletrocardiográficas em chagásicos (informações preliminares sobre o inquérito eletrocardiográfico nacional). Arquivos Brasileiros de Cardiologia 38: 261-264, 1982.

41. Maguire JH, Mott KE, Hoff R, Guimarães A, Franca JT, Souza JAA, Ramos NB, Sherlock IA. A three-year follow-up study of infection with Trypanosoma cruzi and electrocardiographic abnormalities in a rural community 
in Northeast Brazil. American Journal of Tropical Medicine and Hygiene 31: 42-47, 1982.

42. Maguire JH, Mott KE, Lehman JS, Hoff R, Muniz TM, Guimarães AC, Sherlock IA, Morrow RH. Relationship of electrocardiographic abnormalities and seropositivity to Trypanosoma cruz $i$ in a rural community in Northeast Brazil. American Heart Journal 105: 287-294, 1983.

43. New York Heart Association (NYHA). Nomenclature and criteria for diagnosis of disease of the heart and great vessels. 7th ed. Little and Brow Company, Boston, 1973.

44. Organização Mundial da Saúde - Organização Panamericana da Saúde (OMS/OPS). Aspectos de la enfermedad de Chagas. Informe de una reunión conjunta OMS/OPS de investigadores. Boletin de la Oficina Sanitária Panamericana 76: 141-185, 1974.

45. Pereira JB, Coura JR. Aspectos da transmissão da doença de Chagas no município de Virgem da Lapa, Minas Gerais. In: Resumo XIX Congresso da Sociedade Brasileira de Medicina Tropical. Rio de Janeiro, p. $19,1983$.

46. Pereira JB, Coura JR. Letalidade pela doença de Chagas em uma área endèmica com transmissão intertompida. In: Resumo XIX Congresso da Sociedade Brasileira de Medicina Tropical, Rio de Janeiro, p. 20, 1983.

47. Prata A. Prognóstico e complicações da doença de Chagas. Revista Goiana de Medicina 5: 87-96, 1959.

48. Prata A. Natural history of chagasic cardiomyopathy. In: American Trypanosomiasis Research. Symposium PAHO/WHO. Scientific Publication no 318, Washington, p. 191-193, 1976.

49. Pondé A. A cardiopatia crônica da doença de Chagas. Revista Goiana de Medicina 1: 27-70, 1948.

50. Porto CC. Etiologia das cardiopatias em Araguari (Triângulo Mineiro). Estudo da influència das condiçōes sócio-económicas e culturais. Arquivos Brasileiros de Cardiologia 15: 59-68, 1962.

51. Porto CC. O eletrocardiograma no prognóstico e evolu- ção da doença de Chagas. Arquivos Brasileiros de Cardiologia 17: 313-346, 1964.

52. Puigbó JJ, Rhode JRN, Barrios HG, Yepez CG. Clinical and epidemiological study of chronic heart involvement in Chagas'disease. Bulletin World Health Organization 34: 655-669, 1966.

53. Puigbó JJ, Rhode JRN, Barrios HG, Yepez CG. Cuatro años de estudio longitudinal de una comunidad rural con endemicidad chagasica. Boletin de la Oficina Sanitaria Panamericana 66: 112-120, 1969.

54. Rassi A, Carneiro O. Estudo clínico, eletrocardiográfico e radiológico da cardiopatia chagásica crônica. Revista Goiana de Medicina 2: 287-296, 1956.

55. Rezende JM, Rassi A. Comprometimento do esôfago na moléstia de Chagas. Megaesôfago e cardiopatia. Hospital 53: 1-15, 1958.

56. Rezende JM, Oliveira R, Lauar KM. Aspectos clínicos e radiológicos da aperistalsis do esôfago. Revista Brasileira de Gastrenterologia 12: 247-262, 1960.

57. Sheps MC. An examination of some methods of comparing several rates of proportions. Biometrics 15: 87-97, 1959.

58. Souza SL, Camargo ME. The use of filter paper blood smears in a practical fluorescent test for American trypanosomiasis serodiagnosis. Revista do Instituto de Medicina Tropical de São Paulo 8: 255-258, 1966.

59. Torres MCA. Estudo do miocárdio na moléstia de Chagas (forma aguda). Alterações das fibras musculares cardiacas. Memórias do Instituto Oswaldo Cruz 9: 114-139, 1917.

60. Torres CAM. Tripanosomiase americana e a sua anatomia patológica. Folha Médica 4: 259, 1923.

61. Viana G. Contribuição para o estudo da anatomia patológica da "Moléstia de Carlos Chagas" (Esquizotripanoze humana ou tireodite parazitaria). Memórias do Instituto Oswaldo Cruz 3: 276-296, 1911. 